\title{
Better Brain and Cognition Prior to Surgery Is Associated With Elevated Postoperative Brain Extracellular Free-Water in Older Adults
}

\author{
Jared J. Tanner ${ }^{*}$, Manish Amin ${ }^{2}$, Cheshire Hardcastle ${ }^{1}$, Hari Parvataneni ${ }^{3}$, \\ David E. Vaillancourt ${ }^{4}$, Thomas H. Mareci ${ }^{5}$ and Catherine C. Price ${ }^{1,6 *}$
}

${ }^{1}$ Department of Clinical and Health Psychology, University of Florida, Gainesville, FL, United States, ${ }^{2}$ Department of Physics, University of Florida, Gainesville, FL, United States, ${ }^{3}$ Department of Orthopedic Surgery, University of Florida, Gainesville, FL, United States, ${ }^{4}$ Department of Applied Physiology and Kinesiology, Biomedical Engineering, and Neurology, University of Florida, Gainesville, FL, United States, ${ }^{5}$ Department of Biochemistry and Molecular Biology, University of Florida, Gainesville, FL, United States, ${ }^{6}$ Department of Anesthesiology, University of Florida, Gainesville, FL, United States

\section{OPEN ACCESS}

Edited by:

Allison B. Reiss,

Winthrop University Hospital,

United States

Reviewed by:

Brianne M. Bettcher,

University of Colorado Anschutz

Medical Campus, United States

Nan-kuei Chen,

The University of Arizona,

United States

*Correspondence:

Jared J. Tanner

jjtanner@phhp.ufl.edu

Catherine C. Price

cep23@phhp.ufl.edu

Received: 06 November 2018 Accepted: 01 May 2019

Published: 16 May 2019

Citation:

Tanner JJ, Amin M, Hardcastle C

Parvataneni $H$, Vaillancourt $D E$, Mareci TH and Price CC (2019) Better Brain and Cognition Prior to Surgery

Is Associated With Elevated Postoperative Brain Extracellular

Free-Water in Older Adults.

Front. Aging Neurosci. 11:117.

doi: 10.3389/fnagi.2019.00117
For adults age 65 and older, the brain shows acute functional connectivity decreases after total knee arthroplasty with the severity of change predicted by preoperative cognitive function and brain disease burden. The extent of acute structural microstructural brain changes acutely after surgery remains unknown within the literature. For the current study, we report on the severity of acute post-surgery microstructural brain changes as measured by diffusion imaging and free-water analysis. Participants who underwent total knee arthroplasty under general anesthesia and nonsurgery peers were part of a federally funded prospective cohort investigation involving participants. Recruitment occurred between 2013 and 2017. Data were collected in outpatient and inpatient settings within a university-affiliated medical center. A total of 232 TKA patients were referred by the study surgeon and contacted for study inclusion. Of these, 78 met inclusion and exclusion criteria and completed assessment. Five participants were excluded due to anesthetic protocol changes (spinal instead of general) with an additional 12 excluded for imaging-related complications. The total included sample size was 61. A total of 127 non-surgery participants were screened with 66 enrolled. One non-surgery participant was excluded for an imagingrelated complication. Total knee arthroplasty and general anesthetic protocols were standardized. Participants received preoperative neurocognitive assessment and brain magnetic resonance imaging, with repeat imaging $48 \mathrm{~h}$ after surgery or pseudo surgery. Free-water analyses were performed using diffusion weighted images and tract-based spatial statistics with baseline cognitive data used to predict free-water changes. Surgery participants had widespread increases in white matter free-water. Surgery participants with higher cognitive functions as measured by immediate memory and less evidence of brain atrophy and disease (i.e., brain integrity) had greater 
free-water increase. Non-surgery peers had no free-water change. We interpret the surgery group's free-water change as indicating widespread brain white matter glial response, with greater change indicative of better brain response to the acute surgery/anesthesia experience.

Keywords: diffusion magnetic resonance imaging, cognition, brain, neuroinflammation, total knee arthroplasty, anesthesia

\section{INTRODUCTION}

Coupled with the exponential growth of older adults is an increased need or desire for operative interventions that include anesthesia. This is concerning for two reasons: (1) Older age is a risk factor for negative postoperative outcomes (Moller et al., 1998; Monk et al., 2008) and (2) mechanisms for negative outcomes remain poorly understood. Based on integrated theories of brain reserve and diathesis stress models (Belsky and Pluess, 2009), some researchers postulate that patients' preoperative cognitive and brain integrity are important considerations to understanding mechanisms of vulnerability (Goto et al., 2001; Price et al., 2014, 2017).

While functional MRI network connectivity decline is reported by two separate research teams for cardiac surgery (Browndyke et al., 2017) and orthopedic surgery (Huang et al., 2018), much less information is known about acute microstructural changes in older adult brains after surgery. Diffusion-weighted imaging (DWI) can provide information regarding microstructure. When using the analysis method of free-water imaging, which separates diffusion signals into tissue and extracellular components, it is thought that extracellular free-water volume increases can indicate glial change, including neuroinflammation (Pasternak et al., 2009, 2012, 2015).

The purpose of the present investigation was to examine if free-water brain white matter microstructural changes in a study group occur after the same major surgery with the same anesthesia protocol and if observed changes could be predicted by preoperative brain and cognitive integrity. Using a multimodal, longitudinal imaging protocol with both surgical and control groups, we examined metrics of brain disease burden on free-water change. We hypothesized brain disease burden would explain a portion of free-water change. Disease burden was measured using the severity of leukoaraiosis (LA) as an imaging marker of white matter cerebrovascular disease (Hachinski et al., 1987). Leukoaraiosis is a marker of white matter changes with higher levels associated with reduced executive functions within aging and dementia populations (Price et al., 2012; Gold et al., 2017). Ventricular volume was used as a marker of global white and gray matter atrophy (Apostolova et al., 2013) and entorhinal thickness as a sensitive marker of neurodegenerative processes (Devanand et al., 2007). Neurodegeneration, such as in Alzheimer's disease, is associated with chronic neuroinflammatory processes and glial activation (for a review see Griffin et al., 1998), which in turn might explain increases seen in brain white matter in older adults with amnestic mild cognitive impairment and Alzheimer's disease (Ji et al., 2019). Given the dominance of executive/frontal-subcortical functions and memory declines after non-cardiac surgery (Price et al., 2008), aspects of behavioral frontal-subcortical system functions and memory were also examined as potential predictors of microstructural changes.

\section{MATERIALS AND METHODS}

The University of Florida Institutional Review Board in Gainesville, Florida approved this study. All participants were appropriately informed and signed consents. The study was conducted in accordance to principles of the Declaration of Helsinki.

\section{Participants}

A total of 232 surgery patients were referred by the study surgeon (HP) and contacted for study inclusion. Of these, 92 agreed to consider the study with 78 meeting inclusion and exclusion criteria and completing baseline neuropsychological assessment and MRI. Data from five of the participants were excluded due to anesthetic protocol (spinal instead of general). Data from 12 surgery participants were excluded due to presence of pre-existing silent strokes (1 participant), MRI post-surgery scanner unavailability (2 participants), withdrawal before surgery (1 participant), or incomplete postoperative diffusion data due to pain (8 participants). The total included sample size was 61 .

For non-surgery orthopedic peers, a total of 127 participants with self-reported orthopedic pain were recruited from a local orthopedic clinic, community fliers, and targeted mailings. They were screened with 66 enrolled and completed baseline MRI. Non-surgery participants were selected through a yoked review process to match individual surgery participants on age, education, sex, and ethnicity/race. Non-surgery participants had to abstain from surgery for at least 1 year. Both groups were recruited over the same time frame and were tested and scanned at the same time intervals. One participant had an incomplete diffusion sequence, resulting in a total of 65 who completed the baseline assessment and pre-post pseudo surgery imaging sessions.

The final participant sample consisted of non-demented individuals over the age of 60 who elected unilateral total knee arthroplasty surgery (TKA; $n=61$ ) and non-surgery peers $(n=65)$. Groups matched on age, sex, education years, and race. All participants met the following inclusion/exclusion criteria: (1) aged 60 or older, (2) English as primary language, (3) have osteoarthritis or comparable joint pain, (4) have intact activities of daily living, and (5) have baseline neuropsychological testing unsupportive for dementia criteria per Diagnostic 
and Statistical Manual of Mental Disorders - Fifth Edition (American Psychiatric Association [APS], 2013). Additional exclusion criteria included: any other major surgery within the study timeline, history of head trauma/neurodegenerative illness, documented learning or seizure disorder, less than a sixthgrade education, substance abuse in the last year, major cardiac disease, chronic medical illness known to induce encephalopathy, implantable device precluding an MRI, and an unwillingness to complete the MRI.

Participants completed a phone cognitive screening (Welsh et al., 1993; Cook et al., 2009) and a comprehensive history/systems interview to confirm inclusion/exclusion criteria, followed by an in-person comorbidity rating (Charlson et al., 1987), activities of daily living (Lawton and Brody, 1969), cognitive testing, and brain MRI. The same examiner completed testing for all participants. Trained raters blind to group condition scored all behavioral data. Two neuropsychologists reviewed the baseline data to confirm test scores met the expected ranges for non-demented individuals.

\section{Anesthesia and Surgery Protocol}

Protocols were standardized, with surgery participants receiving intravenous midazolam (1-4 mg) followed by continuous femoral nerve block (CFNB) and single-injection subgluteal sciatic with $20 \mathrm{~mL}$ and $30 \mathrm{~mL}$, respectively, of $0.5 \%$ ropivacaine as a bolus injection. The CFNB was continued with ropivacaine $0.2 \%$ at an infusion rate of $10 \mathrm{~mL}$ per hour. No opioids were added. Propofol, fentanyl, and rocuronium were used for anesthesia induction and intubation. Patients were ventilated with an air/oxygen mixture to maintain an end-tidal carbon dioxide at $35 \pm 5 \mathrm{~mm}$; anesthesia was maintained with inhaled isoflurane and intravenous fentanyl and rocuronium.

Total knee replacement surgery was done in a standard manner for all patients by the same surgeon. A tourniquet was used for all cases set to $250 \mathrm{~mm} \mathrm{Hg}$ and elevated prior to incision and deflated just prior to closure. Bony preparation was done by intramedullary instrumentation for the femoral side and extramedullary for the tibial side. The anterior and posterior cruciate ligaments were sacrificed for all patients and implants were fixed to the bone using bone cement.

Using a published conversion algorithm, morphine equivalent dosages (MED) were calculated for each patient relative to the post-surgery scan (Dowell et al., 2016). The MED was considered potentially active if the most recent dose was within $6 \mathrm{~h}$ prior to the post-surgery MRI. Delirium was assessed $24 \mathrm{~h}$ postoperatively with the Confusion Assessment Method (Inouye et al., 1990). Pain assessment ratings $(0-100 ; 100=$ worst) were acquired during the MRI scan.

\section{MRI Acquisition}

Structural MRI was conducted within 1 week on average before surgery and postoperatively within $48 \mathrm{~h}$ after surgery or a pseudo surgery (for non-surgery peers) date. Participants were scanned at two different time points, one corresponding to before surgery and one corresponding to after surgery using a 3.0 T Siemens Verio system with an 8-channel head coil. T1-weighted and diffusion weighted MRI were acquired at both timepoints and T2 FLAIR acquired only at baseline.

T1-weighted images were acquired with the following parameters: TR: $2500 \mathrm{~ms}$; TE: $3.77 \mathrm{~ms} ; 176$ sagittal $1 \mathrm{~mm}^{3}$ slices, $1 \mathrm{~mm}$ isotropic resolution; $256 \times 256 \times 176$ matrix, 7/8 phase partial Fourier. Fluid attenuated inversion recovery (FLAIR) images were acquired with the following parameters: 176 contiguous slices, $1 \mathrm{~mm}^{3}$ voxels, TR/TE $=6000 / 395 \mathrm{~ms}$. Diffusion weighted images were acquired with the following parameters: $\mathrm{TR} / \mathrm{TE}=17300 / 81 \mathrm{~ms}$, two scans without diffusion weighting, six diffusion gradient directions with $b$-value of $100 \mathrm{~s} / \mathrm{mm}^{2}$ and 64 diffusion gradient directions with $b$-value of $1000 \mathrm{~s} / \mathrm{mm}^{2}$. The diffusion gradients were distributed following a scheme of electrostatic repulsion (Jones et al., 1999). The diffusion-weighted images covered the entire brain with an isotropic resolution of $2.0 \mathrm{~mm}$, field of view (FOV) of $256 \mathrm{~mm} \times 256 \mathrm{~mm}$, and 73 slices.

\section{MRI Data Processing}

Using T1-weighted images, cortical reconstruction and volumetric segmentation was performed with the FreeSurfer image analysis suite (Version 6.0 ${ }^{1}$ ) (Fischl et al., 2002, 2004; Segonne et al., 2004; Fischl, 2012). T1 images were first processed using the cross sectional pipeline and then using the longitudinal FreeSurfer pipeline (Reuter et al., 2012) to calculate ventricle volume, and entorhinal cortex thickness.

\section{Diffusion-Weighted Imaging}

Translational diffusion structural sequences allow for not only anatomical comparisons, but also measurement of free-water, which separates diffusion signals into tissue and extracellular components (Pasternak et al., 2009, 2012, 2015). The diffusion weighted scans were corrected for eddy current distortion using the FMRIB Software Library (FSL) eddy_correct algorithm (Smith et al., 2004; Jenkinson et al., 2012) and gradient vectors rotated after correction. To analyze the changes in water diffusion, a free-water elimination model was used, utilizing an isotropic component for the free water compartment and a single tensor for the more restrictive tissue compartment (Pasternak et al., 2009). The isotropic diffusion was modeled using a free-water diffusivity of $3 \times 10^{-3} \mathrm{~s} / \mathrm{mm}^{2}$, which correlates with CSF diffusion in the brain at body temperature. The anisotropic component was analyzed using FSL's dtifit. Final metrics of interest were: Free-water (FW) and tissue-compartment DTI metrics corrected for free-water: fractional anisotropy (FA), mean diffusivity (MD), radial diffusivity (RD; calculated as the mean of eigenvalues, $\lambda_{2}$ and $\lambda_{3}$ ), axial diffusivity (AD; calculated as eigenvalue $\lambda_{1}$ ), $\lambda_{2}$, and $\lambda_{3}$.

For both the TKA and non-TKA groups, voxelwise statistical analysis of the FA data was carried out using TBSS (TractBased Spatial Statistics; Smith et al., 2006), which is part of FSL. All subjects' FA data were then aligned into a common space using the non-linear registration tool FNIRT (Andersson et al., 2007), which uses a b-spline representation of the registration warp field (Rueckert et al., 1999). Next, the mean FA image was calculated and then thinned to create a mean FA skeleton,

\footnotetext{
${ }^{1}$ http://surfer.nmr.mgh.harvard.edu/
} 
which represents the centers of all tracts common to the group. Each participant's aligned FA data was then projected onto this skeleton and the resulting data fed into voxelwise crosssubject statistics using randomise with 5,000 permutations of the data; a within-group, two-sample paired two-tailed $t$-test was performed, correcting for family-wise errors and applying a threshold-free cluster enhancement. All analyses used scan time of day (Trefler et al., 2016), pain during the MRI, and MED score as covariates. The analyses were then repeated for nonFA images using the script tbss_non_FA. We also performed a post hoc between group (surgery vs. control) TBSS analysis to assess FW differences at pre-surgery and post-surgery.

In order to assess individual-level predictors of change in diffusion metrics showing significant pre-post changes, grouplevel $t$-test statistical maps were thresholded at $p<0.05$ (familywise error corrected) and converted to binary masks. Binary masks from metrics with widespread significant change were then transformed into individual native diffusion space and mean metric values within the masks were exported for predictive analysis in SPSS (IBM, Version 24).

\section{Cognitive and Brain Predictors of Interest \\ Current Brain Integrity (BI)}

Neuroanatomical brain integrity (BI), prior to the surgery, was evaluated using a composite of (1) total ventricular volume, (2) mean left and right entorhinal cortex thickness, and (3) leukoaraiosis (LA) volume. Larger lateral ventricular volume is a risk factor for dementia and has been used in calculations of brain reserve (Carmichael et al., 2007). Left and right volumes (including temporal horns) were summed to create total ventricular volume. For each participant, total ventricle volume was divided by total intracranial volume (FreeSurfer maskvol). The entorhinal cortex is sensitive to neurodegenerative pathology and subsequent cognitive decline (Velayudhan et al., 2013). LA is a term for hyperintense or bright regions within the white matter as seen on T2 magnetic resonance (MR) imaging or hypodense regions on computerized tomography (CT) scans (Hachinski et al., 1987; Black et al., 2009). LA associates with white matter pallor on staining, hyalinosis of the vessels, and atherosclerosis (Jellinger, 2013) and broadly is considered a marker of cerebrovascular disease (Black et al., 2009). Increasing amounts of LA are associated with cognitive declines in healthy non-demented older adults and individuals with dementia (Brickman et al., 2010, 2012; Price et al., 2012).

\section{LA Using T2 FLAIR}

A reliable rater measured all scans for LA using FLAIR sequences with in-house macros for Image $J^{2}$. These semiautomated measurements included periventricular caps and rims and showed criterion validity relative to the Junque LA Scale (Price et al., 2012). LA voxels for each brain slice were thresholded and saved as 2D LA binary masks that were then concatenated into individual 3D binary masks (dice similarity coefficient; DSC $=0.84-0.93$; Inter-rater range $=0.80-0.83$; DSC

\footnotetext{
${ }^{2}$ https://imagej.nih.gov
}

mean \pm s.d. $=0.84 \pm 0.12$ ). Outcome variable $=$ Estimated total brain LA volume in $\mathrm{mm}^{3}$.

To create the current BI composite, we calculated $Z$-scores based on all surgery and non-surgery participants. The ventricle and LA $Z$-scores were then multiplied by -1 , resulting in higher $Z$-scores implying a more intact brain. The three $Z$-scores were averaged creating the BI composite variable.

\section{Premorbid Cognitive Reserve}

All baseline measures were acquired within 1 week prior to the surgery/pseudo surgery. Cognitive reserve (CR) is a concept developed to partially explain why there is not a direct relationship between clinical symptoms (e.g., memory) and some factor (e.g., neurodegenerative pathology) that should affect function; higher cognitive reserve is related to psychosocial and experiential factors (e.g., greater educational attainment) and genetic factors (e.g., childhood intelligence) (Stern, 2009). Premorbid cognitive reserve was evaluated as a global composite of three metrics shown to be resistant to neurodegenerative disease processes and are considered estimates of premorbid intelligence (Lezak, 2012): word reading ability [Wide Range Achievement Test (Wilkinson and Robertson, 2006); total words correctly read], vocabulary knowledge [Wechsler Abbreviated Scale of Intelligence; Holdnack (1999); WASI; Vocabulary Subtest], and years of education. We hypothesized CR would explain a portion of free-water changes.

\section{Preoperative Cognitive Domains of Interest}

We completed a comprehensive cognitive assessment to address the a priori hypothesis that preoperative frontal-subcortical functions (e.g., working memory and immediate recall) would explain a portion of pre- to post-surgery structural changes. We tested hypotheses regarding frontal lobe mediated behavior (attention, working memory, and immediate recall) versus temporal lobe mediated behavior (retention and delay recall with relatively weaker working memory and attention components). However, both composites involve measures assessing wide brain networks of bilateral white and gray matter (Jeneson and Squire, 2012) so both composite scores might relate to any white matter changes. The composites are described below.

\section{Frontal-subcortical functions}

Hopkins verbal learning test - revised (HVLT) (Brandt and Benedict, 2001) trial 1 total words recalled, Rey-Osterrieth complex figure (ROCF) (Osterrieth, 1944) immediate recall total score, and digit span backward (Wechsler adult intelligence scale - III) (Holdnack, 1997a), longest backward span. The ROCF immediate recall was performed without a delay after the copy condition.

\section{Delay memory (DM)}

Logical Memory II (delay recall, retention, and recognition scores) from the Wechsler memory scale, 3rd edition (WMSIII) (Holdnack, 1997b), Hopkins verbal learning test - revised (HVLT) delay recall, retention, and recognition (Benedict et al., 1998), and ROCF delay recall total score (Osterrieth, 1944). 


\section{Statistical Analyses}

We assessed between-group differences in age, education, premorbid estimated intelligence, days between MRI scans, general cognition (MoCA), and pain during the MRI using $t$-tests. To assess microstructural white matter changes, we used Pearson product moment correlations to examine associations between cognitive and brain free-water and DTI independent variables of interest. We then used a hierarchical linear regression analysis to examine the combined and proportional variance in free-water change explained by brain integrity, cognitive reserve, frontal-subcortical functions, and declarative memory. Age and race are both associated with systemic inflammation (Lynch, 2010; Stepanikova et al., 2017) so their effects were controlled for in the regression models to account for possible interactions with free-water change. Age and race were entered as Model 1, cognitive reserve was added for Model 2, brain integrity was added for Model 3, frontal-subcortical functions was added for Model 4, and delay memory was added for Model 5. This approach was repeated for $\mathrm{MD}, \mathrm{AD}$, and $\mathrm{RD}$ dependent variables.

\section{RESULTS}

\section{Participant Characteristics}

The final groups of participants (surgery $n=61$; non-surgery $n=65$ ) were not statistically different in age or years of education (Table 1). There was no statistical group difference in baseline pre-surgery pain level while in the MRI scanner; however, post-surgery comparisons showed the surgery group had significantly higher pain than non-surgery peers $(p<0.05)$. Days between baseline/pre-surgery MRI and post-surgery MRI were not statistically different by group $(p=0.18)$. Although four surgery participants were identified with delirium lasting less than one day no participants had evidence of delirium at the time of the post-surgery MRI; all participants were included in analyses. Surgery participants had lower cognitive reserve, frontal-subcortical functions, and delay memory than control participants ( $p$-values $<0.05)$ but did not differ in brain integrity (Table 1). One surgery participant had an acute, focal right inferior parietal white matter stroke that appeared on the post-surgery MRI. There were no reported symptoms with the stroke.

\section{Neuroimaging \\ Free-Water and DTI Analyses}

When comparing post-surgery to pre-surgery, the TKA group had significant widespread increase in FW, $\mathrm{MD}, \mathrm{AD}\left(\lambda_{1}\right), \mathrm{RD}$, $\lambda_{2}$, and $\lambda_{3}$ (family-wise error corrected $p<0.05$; Figure 1). There were no significant differences in FA in the TKA group. Non-TKA peers demonstrate no pre-post differences in any metric. As a post hoc analysis, we performed between-group TBSS analyses at each timepoint. There were no pre-surgery baseline differences in FW or any DTI metric. At the postsurgery timepoint, the TKA group had higher FW than the non-TKA group with the widespread pattern similar to the within-TKA group analyses. The primary metric of interest for individual-level analyses is FW but $\mathrm{MD}, \mathrm{AD}$, and $\mathrm{RD}$ were also assessed to investigate relationship between baseline participant characteristics and perioperative white matter structural changes. The individual with the acute stroke was not an outlier on any of the metrics (all values were within the interquartile range of the post-surgery group).

\section{Cognition and Brain Predictors of Free-Water Change}

Age had a positive association with pre-surgical FW ( $p=0.034)$ but no significant relationships with race or any other freewater or DTI metrics (Table 2). Race did not associate with any independent predictors. Other relationships between presurgical free-water and associated metrics and metric changes are presented in Table 2.

The results of the hierarchical regression predicting freewater change from cognitive and brain variables are presented in Table 3. Frontal-subcortical function was the only significant predictor ( $p=0.01$; medium effect size) of free-water change with current brain integrity (BI) suggestive at a trend level $(p=0.07$; small effect size); higher scores resulted in greater free-water increase after surgery. Age, race, cognitive reserve (CR), and delay memory (DM) were not significant predictors of free-water change ( $p$-values $>0.179)$. These regression models did not significantly predict MD change $(F=1.32, p=0.266)$, AD change $(F=0.57, p=0.750)$, or RD change $(F=1.16, p=0.341)$.

\section{DISCUSSION}

Free-water imaging, a type of DWI analysis, can provide information regarding water diffusivity in tissue and extracellular components. It is proposed extracellular free-water volume increases with neuroinflammation (Pasternak et al., 2009, 2012, 2015), although it could increase due to other factors such as neurodegeneration (Ofori et al., 2015). Within $48 \mathrm{~h}$ of surgery and anesthesia, TKA participants' white matter showed increases in free-water, mean diffusivity, axial diffusivity, and radial diffusivity. The free-water findings indicate increased white matter extracellular volume. We speculate this finding represents widespread neuroinflammation. Axonal compression and decreased axonal diameters driven by increased extracellular volume could explain the $\mathrm{MD}$ and $\mathrm{AD}$ findings. It is possible, however, that $\mathrm{MD}, \mathrm{AD}$, and $\mathrm{RD}$ changes were secondary effects or artifacts of free-water modeling algorithms. Even though we demonstrated $\mathrm{MD}, \mathrm{AD}$, and $\mathrm{RD}$ changes, the absence of FA changes suggests little or no acute neuronal damage.

Importantly, we show individuals with higher frontalsubcortical functions and brain integrity prior to TKA with general anesthesia had greater increases in free-water throughout the core brain white matter. Taken together, while the specific etiology of free-water increases is unknown, a possible explanation for the results is an active cerebral inflammatory response. If the free-water increase represents an active cerebral inflammatory response, individuals with higher preoperative cognition and greater brain integrity appear to have more 
TABLE 1 | Demographic, brain, and cognitive scores by group.

\begin{tabular}{lcc}
\hline & $\begin{array}{c}\text { TKA } \\
(\boldsymbol{n}=\mathbf{6 1 )}\end{array}$ & $\begin{array}{c}\text { Non-TKA } \\
(\boldsymbol{n}=\mathbf{6 5})\end{array}$ \\
\hline Age & $69.13 \pm 7.02,60-85$ & $68.63 \pm 5.59,60-83$ \\
Education & $15.36 \pm 2.85,10-23$ & $16.05 \pm 2.65,9-24$ \\
Race (Black:White) & $8: 53$ & $4: 61$ \\
PreMRI pain & $10.78 \pm 18.14,0-75$ & $8.12 \pm 15.47,0-70$ \\
PostMRI pain** & $38.67 \pm 23.53,0-100$ & $7.60 \pm 10.27,0-40$ \\
MED & $11.97 \pm 10.99,0-37.50$ & - \\
Brain integrity & $-0.05 \pm 0.74,-2.05-1.07$ & $0.04 \pm 0.62,-1.79-1.14$ \\
Cognitive reserve** & $0.34 \pm 0.63,-0.84-1.84$ & $0.69 \pm 0.56,-1.20-1.88$ \\
Processing speed** & $-0.04 \pm 0.54,-1.27-1.29$ & $0.26 \pm 0.68,-1.74-1.84$ \\
Immediate memory** & $-0.36 \pm 0.74,-1.90-1.20$ & $0.10 \pm 0.88,-1.65-2.02$ \\
Delay memory** & $-0.21 \pm 0.79,-2.00-1.06$ & $0.21 \pm 0.71,-2.17-1.40$
\end{tabular}

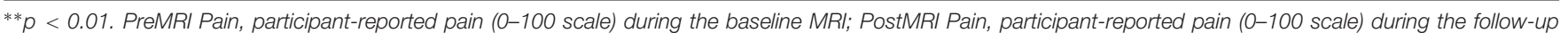

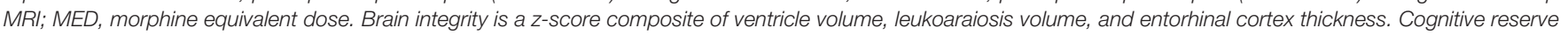
is a composite of word reading ability, vocabulary knowledge, and years of education. Processing speed is a composite of stroop word reading total correct in 45 s, digit symbol raw score in $120 \mathrm{~s}$, trail making test, Part A total time in seconds. Immediate memory is a composite of Hopkins verbal learning test - revised (HVLT) trial 1

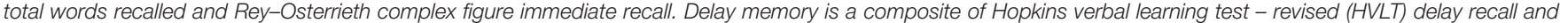
retention and Rey-Osterrieth complex figure delay recall.

resilience to cope with acute cerebral insult via immunological mechanisms. If there is a dominant insulting agent remains an unknown, but we hypothesize the combined effects of anesthetic agents, altered cerebral perfusion, acute pain, surgical stress, and other perioperative and postoperative factors all contribute to extracellular white matter changes.

\section{Chronic Versus Reactive Inflammation?}

One possible interpretation of these results is a responsive glial activation, an acute inflammatory state distinct from - if not completely independent from - chronic neuroinflammation or neurodegeneration. Chronic neuroinflammation is linked with Alzheimer's disease (AD), Parkinson's disease, and multiple sclerosis (MS) (see for review Heneka et al., 2015; Ransohoff, 2016). In AD, for example, astrocytes and microglia appear to interact in a complex way with neuropathology, blood-brain barrier disruption, or other systemic changes in the parenchymal environment (Heneka et al., 2015). While the deleterious effects of chronic inflammation in the brain are becoming clearer, in contrast, less is known about active (reactive) inflammatory states in the human brain, particularly in the context of major surgery.

Notably, non-demented older adults with mild current cognitive inefficiencies and reduced brain integrity have restricted neuroimaging free-water change (Reas et al., 2018). This research suggests cognitive and brain integrity may predict glial response. If free-water increase is a marker for reactive inflammation (glial response), then individuals with reduced preoperative cognitive and brain integrity electing surgery may have a muted response and possibly reduced cerebral "wound" care.

Previous research indicates reactive glial states might be harmful, neutral, or beneficial depending on location, severity, and chronicity (Ransohoff, 2016). There is in vitro evidence pentobarbital results in swollen, "active" microglia (Kannan et al., 2017). It is possible other anesthetics similarly affect glial cells,

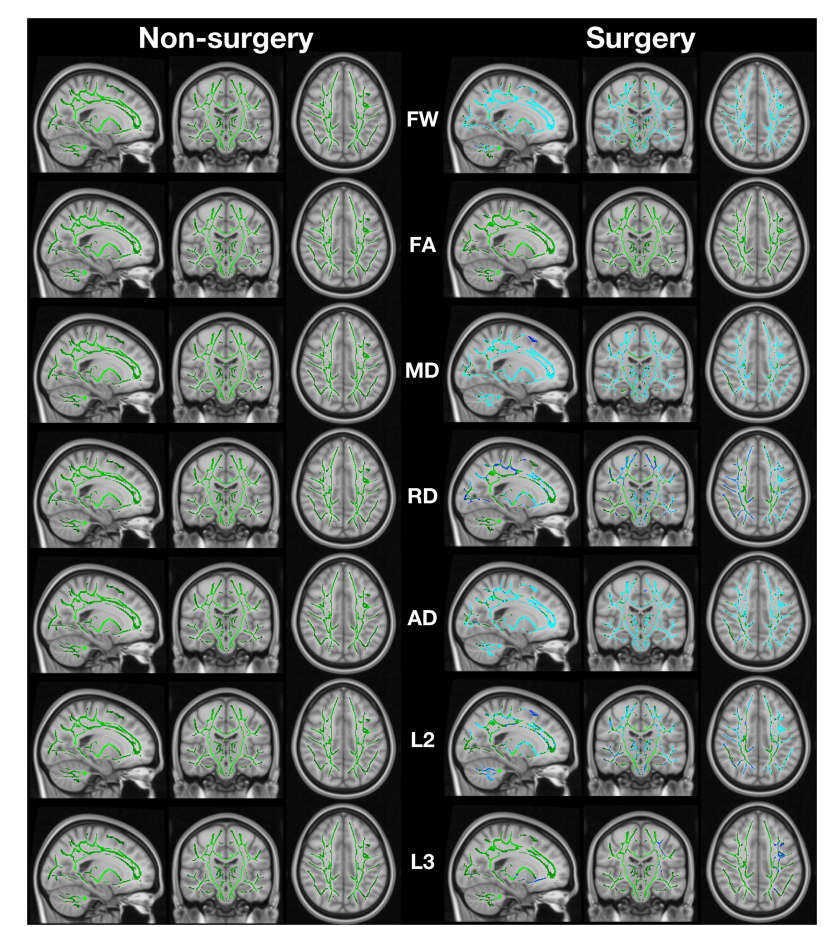

FIGURE 1 | Image shows regions of change in fractional anisotropy (FA), mean diffusivity (MD), radial diffusivity (RD; calculated as the mean of $\lambda_{2}$ and $\left.\lambda_{3}\right)$, axial diffusivity (AD; calculated as $\left.\lambda_{1}\right), \lambda_{2}(L 2)$, and $\lambda_{3}(L 3)$. The green regions are the white matter skeleton created by TBSS. Blue indicates regions where post $>$ pre. Non-surgery participants had no areas of significant change while surgery participants had extensive change (FSL randomise paired $t$-test with 5000 permutations).

creating or adding to a reactive neural inflammatory state. However, in TBI, propofol appears to limit microglial activation, which might serve a neuroprotective function for cognition 
TABLE 2 | Correlations among and descriptive statistics for demographic and dependent variables.

\begin{tabular}{|c|c|c|c|c|c|c|c|c|c|c|}
\hline & Age & Race & PreFW & PreMD & PreAD & PreRD & $\begin{array}{c}\text { Post-Pre } \\
\text { FW }\end{array}$ & $\begin{array}{c}\text { Post-Pre } \\
\text { MD }\end{array}$ & $\begin{array}{c}\text { Post-Pre } \\
\text { AD }\end{array}$ & $\begin{array}{c}\text { Post-Pre } \\
\text { RD }\end{array}$ \\
\hline Age & 1 & 0.03 & $0.27^{*}$ & 0.16 & 0.22 & 0.04 & 0.02 & -0.12 & -0.08 & -0.09 \\
\hline Race & & 1 & -0.13 & -0.11 & -0.13 & -0.06 & 0.11 & 0.09 & -0.04 & 0.09 \\
\hline PreFW & & & 1 & $0.79^{* *}$ & $0.33^{* *}$ & $0.76^{* *}$ & -0.23 & $-0.48^{* *}$ & $-0.34^{* *}$ & $-0.44^{* *}$ \\
\hline PreMD & & & & 1 & $0.58^{* *}$ & $0.92^{* *}$ & -0.19 & $-0.68^{* *}$ & $-0.43^{* *}$ & $-0.63^{* *}$ \\
\hline PreAD & & & & & 1 & 0.24 & 0.06 & $-0.33^{* *}$ & $-0.60^{* *}$ & -0.13 \\
\hline PreRD & & & & & & 1 & -0.24 & $-0.65^{* *}$ & -0.24 & $-0.70^{* *}$ \\
\hline Post-Pre FW & & & & & & & 1 & $0.65^{* *}$ & 0.22 & 0.63 \\
\hline Post-Pre MD & & & & & & & & 1 & $0.57^{* *}$ & $0.90^{* *}$ \\
\hline Post-Pre AD & & & & & & & & & 1 & 0.19 \\
\hline Post-Pre RD & & & & & & & & & & 1 \\
\hline
\end{tabular}

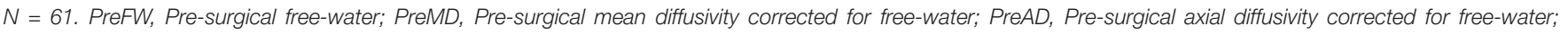

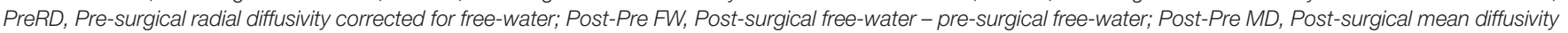

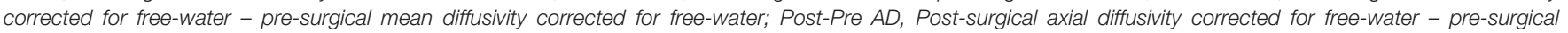
axial diffusivity corrected for free-water; Post-Pre RD, Post-surgical radial diffusivity corrected for free-water - pre-surgical radial diffusivity corrected for free-water. ${ }^{*} p<0.05 .{ }^{* *} p<0.01$.

(Luo et al., 2013). Similarly, in animal models of surgery, elevated interleukin-1 $\beta$ (IL-1 $\beta$ ) is associated with memory dysfunction; pretreatment with an IL-1 receptor antagonist reduced neuroinflammatory effects and memory dysfunction (Cibelli et al., 2010). In contrast, however, it was shown in $\mathrm{AD}$ that greater baseline (acute/reactive) microglial activation as measured through ${ }^{18} \mathrm{~F}-\mathrm{DPA}-714$ binding was associated with better clinical prognosis 2 years later. Individuals with lower baseline microglial activation subsequently had higher (chronic) microglial activation and worse clinical outcome (Hamelin et al., 2018). While we do not know if the freewater changes in the context of major surgery indicate beneficial neuroinflammation, or are specific to inflammation, it is suggestive that those with worse cognition and lower brain integrity have a muted perioperative response. We speculate those who have muted responses are less able to return to free-water baseline and the acute inflammation increases chronic inflammation. Additionally, those who have worse baseline cognition and brain integrity are at increased risk of adverse outcomes after major surgery (Chen et al., 2014; Price et al., 2014; Culley et al., 2017) so subdued acute postoperative neuroinflammation might be a mechanism for postoperative cognitive changes. This is an area for future investigation.

Patients with mild cognitive impairment (MCI) who are electing surgery might have chronic inflammation and altered (senescent) microglia, thereby resulting in a restricted ability to respond to neural insults associated with surgery and general anesthesia. While we did not analyze MCI, it is possible MCI could partially explain the lower levels of free-water increase after surgery. Importantly, our results also suggest a potential mechanism for neurodegeneration in the future. If major surgery under general anesthesia acts as a mild but widespread brain trauma with associated innate immune response, it is possible the initial healing response will interact with existing neuropathology, weakened blood-brain barriers, and chronic inflammation to result in future additional pathological burden and neurodegeneration. Research suggests undergoing anesthesia and surgery result in increased risk of dementia and reduced time to dementia diagnosis (Chen et al., 2014). However, a study with 22 female patients using cerebrospinal fluid markers of inflammation indicated that while a neuroinflammatory response occurs there were not significant beta-amyloid or tau biomarker changes (Pikwer et al., 2017). Those results were preliminary and do not rule out a subtle longitudinal cascading effect on neuropathology. They do, however, support an interpretation of our results as indicating a reactive glial state following surgery.

Hirsch et al. (2016) assessed cerebrospinal fluid (CSF) and plasma inflammatory markers after orthopedic surgery in a pilot group of 11 older patients. They found increased pro- and anti-inflammatory activity in the central nervous system after surgery. Intriguingly, Hirsch et al. (2016) had preliminary data suggesting patients with acute postoperative cognitive decline had lower preoperative levels of some pro and anti-inflammatory plasma cytokines with some cytokines continuing to remain lower after surgery. In contrast, some CSF cytokines were higher preoperatively and remained higher postoperatively in patients who developed cognitive difficulties acutely after surgery. The mixed results are likely cytokine-specific and could relate to timing of measurements. In other words, reactive glial states might be harmful, neutral, or beneficial depending on a number of factors including how, what, and when measurements are taken (Ransohoff, 2016).

\section{Glymphatic System}

An alternative but not necessarily competing explanation for free-water increases relates to the glymphatic system - a system believed to play a role in the clearance of cerebral waste (Iliff and Nedergaard, 2013). Neural injury results in increased generation of metabolic byproducts. Major surgery under general anesthesia might result in transient neural injury, at least for older adults. There would then be a need for the glymphatic 


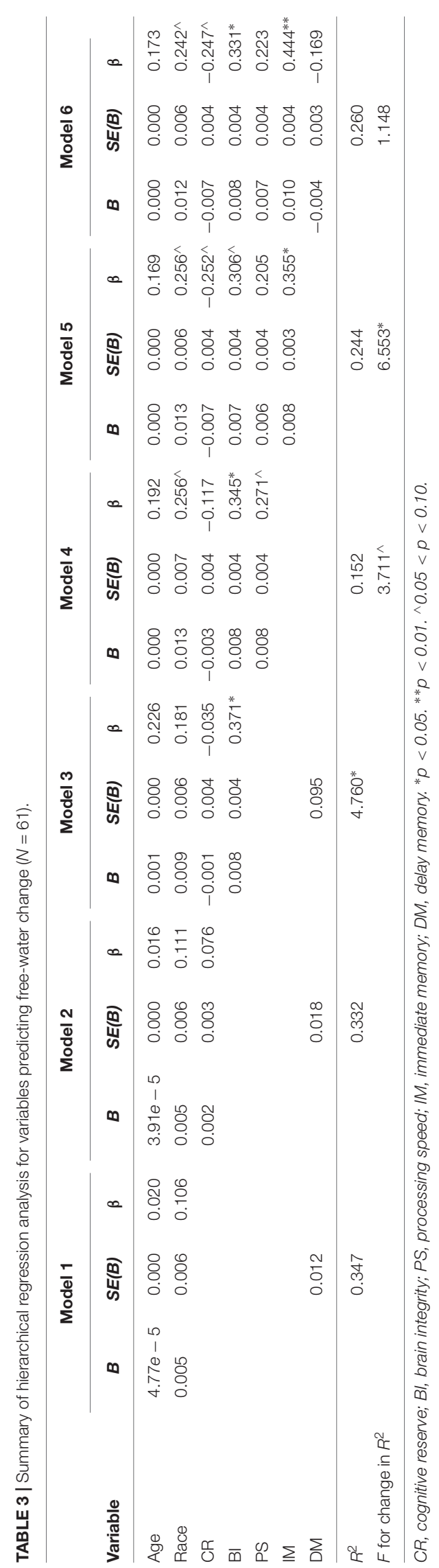

system to activate and clear excessive metabolic byproducts. Evidence suggests the glymphatic system is more active during sleep and under general anesthesia (Xie et al., 2013). During sleep and under anesthesia there is increased interstitial fluid volume associated with activation of the glymphatic system. It is believed sleep might thus serve in part as a restorative function to remove accumulation of potentially neurotoxic waste products (Xie et al., 2013).

Apart from sleep, if general anesthesia also results in increased interstitial fluid volume (Xie et al., 2013), increased free-water in our participants could be caused by increased interstitial fluid volume secondary to general anesthesia. The relationship between interstitial fluid volume and anesthesia is not clear, however, in light of contradictory findings by Gakuba et al. (2018) who did not find increases in extracellular water volume in anesthetized mice.

There is indirect evidence in favor of a glymphatic hypothesis for why patients with reduced cognitive function may have less pre-post microstructural free-water change. Tarasoff-Conway et al. (2015) provide a review of research implications of brain clearance systems for Alzheimer's disease. Aqp4 knockout mouse models demonstrate reduced interstitial and $A \beta$ clearance. Additionally, mouse models of traumatic brain injury suggest impaired glymphatic function for at least one-month post-injury (Iliff et al., 2014). Individuals with accumulating $A \beta$ and disrupted interstitial clearance, which might be the case in individuals who have prodromal Alzheimer's disease, thus might be less likely to have a responsive glymphatic system and less extracellular volume increase (Kress et al., 2014).

While the etiology of the free-water increases is unknown, we observed extensive acute white matter free-water changes after major orthopedic surgery. Of the two explanations explored, both suggest glial involvement. There are other possibilities, but there appears to be a widespread glial activation after major TKA with general anesthesia. While most participants experienced free-water increase, those who had greater immediate memory and brain integrity had relatively greater acute perioperative increases. Therefore, if the freewater change represents glial activation, our results suggest the reactive glial response is greater in individuals with higher scores on preoperative measures of immediate memory and brain integrity. Whether or not free-water change has longitudinal benefits for cognition and recovery is under investigation. Remaining unknowns also include (1) the specific etiology of changes, (2) if diffusion changes persist after the acute postoperative stage, (3) if acute white matter changes are predictive of other later adverse events, and (4) if mild cognitive impairment as a classification is predictive of restricted freewater changes.

\section{Other Considerations}

There are other considerations for etiology behind extracellular free-water changes. Widespread edema secondary to blood brain barrier disruption is a possibility; cerebral edema has been shown acutely after major cardiac surgery (Harris et al., 1993; Anderson et al., 1999). This could be an area of focus in 
future analyses of the etiology of extracellular free-water increases after major surgery.

One of the components of our brain integrity composite was leukoaraiosis, which is associated with cerebrovascular and cardiovascular disease. Cardiovascular disease might play a mechanistic role in free-water change after surgery. However, in a post hoc analysis found no significant correlations between FW change and other cardiovascular study variables (i.e., systolic and diastolic blood pressure, pulse pressure, cholesterol, triglycerides, HDL, LDL, self-reported cardiovascular risk factors, p-values $>0.233$ ).

\section{Limitations}

We did not acquire postoperative IL-6, TNF-alpha, CRP or other plasma or CSF markers of inflammation. Blood markers, however, are non-specific to neuroinflammation so, while they might provide evidence of inflammation, without corroborating markers of neuroinflammation (e.g., free-water increase) they are not sufficient markers of neuroinflammation. While we are not certain the changes in free-water result from neuroinflammation, free-water change is localized within the brain and are a mechanism and method worth continued investigation. Another limitation is we did not acquire a repeated FLAIR scan to quantify LA changes, which might have yielded additional information about acute white matter changes. We also did not assess free-water changes within gray matter regions. Gray matter free-water change analyses are encouraged in future research.

\section{CONCLUSION}

We demonstrated older adults undergoing TKA under general anesthesia have extensive increases in white matter free-water, suggestive of extracellular volume increases. Importantly, older adults with better frontal-subcortical cognitive functions (e.g., immediate memory) and better brain integrity had relatively larger increases in free-water. Our results suggest an active white matter glial response (e.g., neuroinflammation and/or glymphatic changes) to surgery under general anesthesia.

\section{REFERENCES}

American Psychiatric Association [APS] (2013). Diagnostic and Statistical Manual of Mental Disorders. Washington, DC: American Psychiatric Publishing.

Anderson, R. E., Li, T. Q., Hindmarsh, T., Settergren, G., and Vaage, J. (1999). Increased extracellular brain water after coronary artery bypass grafting is avoided by off-pump surgery. J. Cardiothora. Vasc. Anesth. 13, 698-702. doi: 10.1016/s1053-0770(99)90123-4

Andersson, J. L., Jenkinson, M., and Smith, S. (2007). Non-Linear Optimisation. FMRIB Technical Report TR07JA1. Oxford: FMRIB Centre.

Apostolova, L. G., Babakchanian, S., Hwang, K. S., Green, A. E., Zlatev, D., Chou, Y. Y., et al. (2013). Ventricular enlargement and its clinical correlates in the imaging cohort from the ADCS MCI donepezil/vitamin E study. Alzheimer Dis. Assoc. Disord. 27, 174-181. doi: 10.1097/WAD.0b013e3182 $677 \mathrm{~b} 3 \mathrm{~d}$

\section{ETHICS STATEMENT}

This study was carried out in accordance with the recommendations of the University of Florida Institutional Review Board in Gainesville, Florida with written informed consent from all subjects. All subjects gave written informed consent in accordance with the Declaration of Helsinki. The protocol was approved by the University of Florida Institutional Review Board.

\section{AUTHOR CONTRIBUTIONS}

CCP, HP, and TM contributed to the design of the research. JT, HP, and CCP contributed to the implementation of the research. JT, MA, CH, DV, TM, and CCP contributed to the analysis or interpretation of the results. JT, MA, HP, DV, and CCP contributed to the writing of the manuscript.

\section{FUNDING}

This work was supported by the National Institutes of Health grant nos. R01 NR014181 (to CCP) and UL1TR001427 (CTSI). The content is solely the responsibility of the authors and does not necessarily represent the official views of the National Institute on Aging or the National Institutes of Health.

\section{ACKNOWLEDGMENTS}

This manuscript would not have been possible without the participants who volunteered their time and effort the current investigation. We are deeply appreciative to them. We acknowledge the work of the graduate students: Loren Hizel, M.S., Samuel Crowley, M.S., Elle Wiggins, M.S., and Nadine Schwab, Ph.D., for their assistance with data acquisition. We acknowledged are the important research coordinators who made this project possible: Donna Weber, B.S. and Kristi Ayers, B.S. We also acknowledge the MR technology staff who graciously allowed us to use the clinical 3T Siemens scanner to complete this important investigation.

Belsky, J., and Pluess, M. (2009). Beyond diathesis stress: differential susceptibility to environmental influences. Psychol. Bull. 135, 885-908. doi: 10.1037/ a0017376

Benedict, R. H. B., Schretlen, D., Groninger, L., and Brandt, J. (1998). Hopkins verbal learning test revised: normative data and analysis of inter-form and test-retest reliability. Clin. Neuropsychol. 12, 43-55. doi: 10.1076/clin.12.1.43. 1726

Black, S., Gao, F., and Bilbao, J. (2009). Understanding white matter disease: imaging-pathological correlations in vascular cognitive impairment. Stroke 40 (3 Suppl.), S48-S52. doi: 10.1161/STROKEAHA.108. 537704

Brandt, J., and Benedict, R. H. (2001). Hopkins Verbal Learning Test-Revised: Professional Manual. Lutz, FL: Psychological Assessment Resources.

Brickman, A. M., Provenzano, F. A., Muraskin, J., Manly, J. J., Blum, S., Apa, Z., et al. (2012). Regional white matter hyperintensity volume, not hippocampal 
atrophy, predicts incident Alzheimer disease in the community. Arch. Neurol. 69, 1621-1627. doi: 10.1001/archneurol.2012.1527

Brickman, A. M., Reitz, C., Luchsinger, J. A., Manly, J. J., Schupf, N., Muraskin, J., et al. (2010). Long-term blood pressure fluctuation and cerebrovascular disease in an elderly cohort. Arch. Neurol. 67, 564-569. doi: 10.1001/archneurol. 2010.70

Browndyke, J. N., Berger, M., Harshbarger, T. B., Smith, P. J., White, W., Bisanar, T. L., et al. (2017). Resting-state functional connectivity and cognition after major cardiac surgery in older adults without preoperative cognitive impairment: preliminary findings. J. Am. Geriatr. Soc. 65, e6-e12. doi: 10.1111/ jgs. 14534

Carmichael, O. T., Kuller, L. H., Lopez, O. L., Thompson, P. M., Dutton, R. A., $\mathrm{Lu}$, A., et al. (2007). Cerebral ventricular changes associated with transitions between normal cognitive function, mild cognitive impairment, and dementia. Alzheimer Dis. Assoc. Disord. 21, 14-24. doi: 10.1097/WAD.0b013e318032d2b1

Charlson, M. E., Pompei, P., Ales, K. L., and MacKenzie, C. R. (1987). A new method of classifying prognostic comorbidity in longitudinal studies: development and validation. J. Chronic Dis. 40, 373-383. doi: 10.1016/00219681(87)90171-8

Chen, C.-W., Lin, C.-C., Chen, K.-B., Kuo, Y.-C., Li, C.-Y., and Chung, C.-J. (2014). Increased risk of dementia in people with previous exposure to general anesthesia: a nationwide population-based case-control study. Alzheimers Dement. 10, 196-204. doi: 10.1016/j.jalz.2013.05.1766

Cibelli, M., Fidalgo, A. R., Terrando, N., Ma, D., Monaco, C., Feldmann, M., et al. (2010). Role of interleukin-1 $\beta$ in postoperative cognitive dysfunction. Ann. Neurol. 68, 360-368.

Cook, S. E., Marsiske, M., and McCoy, K. J. (2009). The use of the modified telephone interview for cognitive status (TICS-M) in the detection of amnestic mild cognitive impairment. J. Geriatr. Psychiatry Neurol. 22, 103-109. doi: $10.1177 / 0891988708328214$

Culley, D. J., Flaherty, D., Fahey, M. C., Rudolph, J. L., Javedan, H., Huang, C. C., et al. (2017). Poor performance on a preoperative cognitive screening test predicts postoperative complications in older orthopedic surgical patients. Anesthesiology 127, 765-774. doi: 10.1097/ALN.0000000000001859

Devanand, D. P., Pradhaban, G., Liu, X., Khandji, A., De Santi, S., Segal, S., et al. (2007). Hippocampal and entorhinal atrophy in mild cognitive impairment: prediction of Alzheimer disease. Neurology 68, 828-836. doi: 10.1212/01.wnl. 0000256697.20968.d7

Dowell, D., Haegerich, T. M., and Chou, R. (2016). Cdc guideline for prescribing opioids for chronic pain-united states, 2016. JAMA 315, 1624-1645. doi: 10. 1001/jama.2016.1464

Fischl, B. (2012). FreeSurfer. NeuroImage 62, 774-781. doi: 10.1016/j.neuroimage. 2012.01.021

Fischl, B., Salat, D. H., Busa, E., Albert, M., Dieterich, M., Haselgrove, C., et al. (2002). Whole brain segmentation: automated labeling of neuroanatomical structures in the human brain. Neuron 33, 341-355.

Fischl, B., Salat, D. H., van der Kouwe, A. J. W., Makris, N., Ségonne, F., Quinn, B. T., et al. (2004). Sequence-independent segmentation of magnetic resonance images. NeuroImage 23, S69-S84. doi: 10.1016/j.neuroimage.2004.07.016

Gakuba, C., Gaberel, T., Goursaud, S., Bourges, J., Di Palma, C., Quenault, A., et al. (2018). General anesthesia inhibits the activity of the "glymphatic system". Theranostics 8, 710-722. doi: 10.7150/thno.19154

Gold, B. T., Brown, C. A., Hakun, J. G., Shaw, L. M., Trojanowski, J. Q., and Smith, C. D. (2017). Clinically silent Alzheimer's and vascular pathologies influence brain networks supporting executive function in healthy older adults. Neurobiol. Aging 58, 102-111. doi: 10.1016/j.neurobiolaging.2017.06.012

Goto, T., Baba, T., Honma, K., Shibata, Y., Arai, Y., Uozumi, H., et al. (2001). Magnetic resonance imaging findings and postoperative neurologic dysfunction in elderly patients undergoing coronary artery bypass grafting. Ann. Thorac. Surg. 72, 137-142. doi: 10.1016/S0003-4975(01)02676-5

Griffin, W. S., Sheng, J. G., Royston, M. C., Gentleman, S. M., McKenzie, J. E., Graham, D. I., et al. (1998). Glial-neuronal interactions in Alzheimer's disease: the potential role of a 'cytokine cycle' in disease progression. Brain Pathol. 8, 65-72. doi: 10.1111/j.1750-3639.1998.tb00136.x

Hachinski, V. C., Potter, P., and Merskey, H. (1987). Leuko-araiosis. Arch. Neurol. 44, 21-23. doi: 10.1001/archneur.1987.00520130013009

Hamelin, L., Lagarde, J., Dorothée, G., Potier, M. C., Corlier, F., Kuhnast, B., et al. (2018). Distinct dynamic profiles of microglial activation are associated with progression of Alzheimer's disease. Brain 141, 1855-1870. doi: 10.1093/brain/ awy079

Harris, D. N. F., Bailey, S. M., Smith, P. L. C., Taylor, K. M., Oatridge, A., and Bydder, G. M. (1993). Brain swelling in first hour after coronary artery bypass surgery. Lancet 342, 586-587. doi: 10.1016/0140-6736(93)91412-f

Heneka, M. T., Carson, M. J., El Khoury, J., Landreth, G. E., Brosseron, F., Feinstein, D. L., et al. (2015). Neuroinflammation in Alzheimer's disease. Lancet Neurol. 14, 388-405.

Hirsch, J., Vacas, S., Terrando, N., Yuan, M., Sands, L. P., Kramer, J., et al. (2016). Perioperative cerebrospinal fluid and plasma inflammatory markers after orthopedic surgery. J. Neuroinflamm. 13:211. doi: 10.1186/s12974-0160681-9

Holdnack, H. A. (1997a). Wechsler Adult Intelligence Scale, (WAIS-III), 3rd Edn. San Antonio, TX: Psychological Corporation.

Holdnack, H. A. (1997b). Wechsler Memory Scale III, 3rd Edn. San Antonio, TX: The Psychological Corporation.

Holdnack, H. A. (1999). Wechsler Abbreviated Scale of Intelligence. San Antonio, TX: Harcourt Assessment.

Huang, H., Tanner, J., Parvataneni, H., Rice, M., Horgas, A., Ding, M., et al. (2018). Impact of total knee arthroplasty with general anesthesia on brain networks: cognitive efficiency and ventricular volume predict functional connectivity decline in older adults. J. Alzheimers Dis. 62, 319-333. doi: 10.3233/JAD170496

Iliff, J. J., Chen, M. J., Plog, B. A., Zeppenfeld, D. M., Soltero, M., Yang, L., et al. (2014). Impairment of glymphatic pathway function promotes tau pathology after traumatic brain injury. J. Neurosci. 34, 16180-16193. doi: 10. 1523/JNEUROSCI.3020-14.2014

Iliff, J. J., and Nedergaard, M. (2013). Is there a cerebral lymphatic system?. Stroke 44(6 Suppl. 1), S93-S95.

Inouye, S. K., van Dyck, C. H., Alessi, C. A., Balkin, S., Siegal, A. P., and Horwitz, R. I. (1990). Clarifying confusion: the confusion assessment method. A new method for detection of delirium. Ann. Intern. Med. 113, 941-948.

Jellinger, K. A. (2013). Pathology and pathogenesis of vascular cognitive impairment-a critical update. Front. Aging Neurosci. 5:17. doi: 10.3389/fnagi. 2013.00017

Jeneson, A., and Squire, L. R. (2012). Working memory, long-term memory, and medial temporal lobe function. Learn. Mem. 19, 15-25. doi: 10.1101/lm.0240 18.111

Jenkinson, M., Beckmann, C. F., Behrens, T. E., Woolrich, M. W., and Smith, S. M. (2012). Fsl. Neuroimage 62, 782-790. doi: 10.1016/j.neuroimage.2011.09.015

Ji, F., Pasternak, O., Ng, K. K., Chong, J. S. X., Liu, S., Zhang, L., et al. (2019). White matter microstructural abnormalities and default network degeneration are associated with early memory deficit in Alzheimer's disease continuum. Sci. Rep. 9:4749.

Jones, D. K., Horsfield, M. A., and Simmons, A. (1999). Optimal strategies for measuring diffusion in anisotropic systems by magnetic resonance imaging. Magn. Reson. Med. 42, 515-525. doi: 10.1002/(sici)1522-2594(199909)42: $3<515::$ aid-mrm14>3.3.co;2-h

Kannan, G., Kambhampati, S. P., and Kudchadkar, S. R. (2017). Effect of anesthetics on microglial activation and nanoparticle uptake: implications for drug delivery in traumatic brain injury. J. Controll. Release 263, 192-199. doi: 10.1016/j. jconrel.2017.03.032

Kress, B. T., Iliff, J. J., Xia, M., Wang, M., Wei, H. S., Zeppenfeld, D., et al. (2014). Impairment of paravascular clearance pathways in the aging brain. Ann. Neurol. 76, 845-861. doi: 10.1002/ana.24271

Lawton, M. P., and Brody, E. M. (1969). Assessment of older people: selfmaintaining and instrumental activities of daily living. Gerontologist 9(3 Pt 1), 179-186. doi: 10.1093/geront/9.3_Part_1.179

Lezak, M. D. (2012). Neuropsychological Assessment. Oxford: Oxford University Press.

Luo, T., Wu, J., Kabadi, S. V., Sabirzhanov, B., Guanciale, K., Hanscom, M., et al. (2013). Propofol limits microglial activation after experimental brain trauma through inhibition of nicotinamide adenine dinucleotide phosphate oxidase. Anesthesiology 119, 1370-1388. doi: 10.1097/ALN.0000000000000020

Lynch, M. A. (2010). Age-related neuroinflammatory changes negatively impact on neuronal function. Front. Aging Neurosci. 1:6. doi: 10.3389/neuro.24.006.2009

Moller, J. T., Cluitmans, P., Rasmussen, L. S., Houx, P., Rasmussen, H., Canet, J., et al. (1998). Long-term postoperative cognitive dysfunction in the elderly 
ISPOCD1 study. ISPOCD investigators. international study of postoperative cognitive dysfunction. Lancet 351, 857-861. doi: 10.1016/s0140-6736(97) 07382-0

Monk, T. G., Weldon, B. C., Garvan, C. W., Dede, D. E., van der Aa, M. T., Heilman, K. M., et al. (2008). Predictors of cognitive dysfunction after major noncardiac surgery. Anesthesiology 108, 18-30. doi: 10.1097/01.anes. 0000296071.19434.1e

Ofori, E., Pasternak, O., Planetta, P. J., Li, H., Burciu, R. G., Snyder, A. F., et al. (2015). Longitudinal changes in free-water within the substantia nigra of Parkinson's disease. Brain 138(Pt 8), 2322-2331. doi: 10.1093/brain/awv136

Osterrieth, P. A. (1944). Le test de copie d'une figure complexe; contribution à l'étude de la perception et de la mémoire. Arch. de Psychol. 30, 206-356.

Pasternak, O., Sochen, N., Gur, Y., Intrator, N., and Assaf, Y. (2009). Free water elimination and mapping from diffusion MRI. Magn. Reson. Med. 62, 717-730. doi: $10.1002 / \mathrm{mrm} .22055$

Pasternak, O., Westin, C. F., Bouix, S., Seidman, L. J., Goldstein, J. M., Woo, T. U., et al. (2012). Excessive extracellular volume reveals a neurodegenerative pattern in schizophrenia onset. J. Neurosci. 32, 17365-17372. doi: 10.1523/ JNEUROSCI.2904-12.2012

Pasternak, O., Westin, C. F., Dahlben, B., Bouix, S., and Kubicki, M. (2015). The extent of diffusion MRI markers of neuroinflammation and white matter deterioration in chronic schizophrenia. Schizophr. Res. 161, 113-118. doi: 10. 1016/j.schres.2014.07.031

Pikwer, A., Castegren, M., Namdar, S., Blennow, K., Zetterberg, H., and Mattsson, N. (2017). Effects of surgery and propofol-remifentanil total intravenous anesthesia on cerebrospinal fluid biomarkers of inflammation, Alzheimer's disease, and neuronal injury in humans: a cohort study. J. Neuroinflamm. 14:193. doi: 10.1186/s12974-017-0950-2

Price, C. C., Garvan, C., Hizel, L. P., Lopez, M. G., and Billings, F. T. (2017). Delayed recall and working memory MMSE domains predict delirium following cardiac surgery. J. Alzheimers Dis. 59, 1027-1035. doi: 10.3233/JAD170380

Price, C. C., Garvan, C. W., and Monk, T. G. (2008). Type and severity of cognitive decline in older adults after noncardiac surgery. Anesthesiology 108, 8-17. doi: 10.1097/01.anes.0000296072.02527.18

Price, C. C., Mitchell, S. M., Brumback, B., Tanner, J. J., Schmalfuss, I., Lamar, M., et al. (2012). MRI-leukoaraiosis thresholds and the phenotypic expression of dementia. Neurology 79, 734-740. doi: 10.1212/WNL.0b013e3182661ef6

Price, C. C., Tanner, J. J., Schmalfuss, I., Garvan, C. W., Gearen, P., Dickey, D., et al. (2014). A pilot study evaluating presurgery neuroanatomical biomarkers for postoperative cognitive decline after total knee arthroplasty in older adults. Anesthesiology 120, 601-613. doi: 10.1097/ALN.0000000000000080

Ransohoff, R. M. (2016). How neuroinflammation contributes to neurodegeneration. Science 353, 777-783. doi: 10.1126/science.aag2590

Reas, E. T., Hagler, D. J. Jr., White, N. S., Kuperman, J. M., Bartsch, H., Wierenga, C. E., et al. (2018). Microstructural brain changes track cognitive decline in mild cognitive impairment. NeuroImage Clin. 20, 883-891. doi: 10.1016/j.nicl.2018. 09.027

Reuter, M., Schmansky, N. J., Rosas, H. D., and Fischl, B. (2012). Within-subject template estimation for unbiased longitudinal image analysis. Neuroimage 61, 1402-1418. doi: 10.1016/j.neuroimage.2012.02.084
Rueckert, D., Sonoda, L. I., Hayes, C., Hill, D. L., Leach, M. O., and Hawkes, D. J. (1999). Nonrigid registration using free-form deformations: application to breast MR images. IEEE Trans. Med. Imaging 18, 712-721. doi: 10.1109/42. 796284

Segonne, F., Dale, A. M., Busa, E., Glessner, M., Salat, D., Hahn, H. K., et al. (2004). A hybrid approach to the skull stripping problem in MRI. Neuroimage 22, 1060-1075. doi: 10.1016/j.neuroimage.2004.03.032

Smith, S. M., Jenkinson, M., Johansen-Berg, H., Rueckert, D., Nichols, T. E., Mackay, C. E., et al. (2006). Tract-based spatial statistics: voxelwise analysis of multi-subject diffusion data. NeuroImage 31, 1487-1505. doi: 10.1016/j. neuroimage.2006.02.024

Smith, S. M., Jenkinson, M., Woolrich, M. W., Beckmann, C. F., Behrens, T. E., Johansen-Berg, H., et al. (2004). Advances in functional and structural MR image analysis and implementation as FSL. Neuroimage 23(Suppl. 1), S208S219. doi: 10.1016/j.neuroimage.2004.07.051

Stepanikova, I., Bateman, L. B., and Oates, G. R. (2017). Systemic inflammation in midlife: race, socioeconomic status, and perceived discrimination. Am. J. Prevent. Med. 52, S63-S76. doi: 10.1016/j.amepre.2016.09.026

Stern, Y. (2009). Cognitive reserve. Neuropsychologia 47, 2015-2028. doi: 10.1016/ j.neuropsychologia.2009.03.004

Tarasoff-Conway, J. M., Carare, R. O., Osorio, R. S., Glodzik, L., Butler, T., Fieremans, E., et al. (2015). Clearance systems in the brain-implications for Alzheimer disease. Nat. Rev. Neurol. 11:457. doi: 10.1038/nrneurol.2015.119

Trefler, A., Sadeghi, N., Thomas, A. G., Pierpaoli, C., Baker, C. I., and Thomas, C. (2016). Impact of time-of-day on brain morphometric measures derived from T 1-weighted magnetic resonance imaging. NeuroImage 133, 41-52. doi: 10.1016/j.neuroimage.2016.02.034

Velayudhan, L., Proitsi, P., Westman, E., Muehlboeck, J., Mecocci, P., Vellas, B., et al. (2013). Entorhinal cortex thickness predicts cognitive decline in Alzheimer's disease. J. Alzheimers Dis. 33, 755-766. doi: 10.3233/jad-2012121408

Welsh, K. A., Breitner, J. C. S., and Magruder-Habib, K. M. (1993). Detection of dementia in the elderly using telephone screening of cognitive status. Cogn. Behav. Neurol. 6, 103-110.

Wilkinson, G. S., and Robertson, G. J. (2006). WRAT 4 : Wide Range Achievement Test; Professional Manual. Lutz, FL: Psychological Assessment Resources, Inc.

Xie, L., Kang, H., Xu, Q., Chen, M. J., Liao, Y., Thiyagarajan, M., et al. (2013). Sleep drives metabolite clearance from the adult brain. Science 342, 373-377. doi: $10.1126 /$ science. 1241224

Conflict of Interest Statement: The authors declare that the research was conducted in the absence of any commercial or financial relationships that could be construed as a potential conflict of interest.

Copyright (c) 2019 Tanner, Amin, Hardcastle, Parvataneni, Vaillancourt, Mareci and Price. This is an open-access article distributed under the terms of the Creative Commons Attribution License (CC BY). The use, distribution or reproduction in other forums is permitted, provided the original author(s) and the copyright owner(s) are credited and that the original publication in this journal is cited, in accordance with accepted academic practice. No use, distribution or reproduction is permitted which does not comply with these terms. 